\title{
microRNA-Based Network and Pathway Analysis for Neuropathic Pain in Rodent Models
}

\author{
Yi-Li Zheng ${ }^{1}$, Xuan Su ${ }^{1}$, Yu-Meng Chen ${ }^{1}$, Jia-Bao Guo ${ }^{2}$, Ge Song ${ }^{3}$, Zheng Yang ${ }^{4}$, \\ Pei-Jie Chen ${ }^{1 *}$ and Xue-Qiang Wang ${ }^{1,5 *}$
}

${ }^{1}$ Department of Sport Rehabilitation, Shanghai University of Sport, Shanghai, China, ${ }^{2}$ The Second School of Clinical Medical, Xuzhou Medical University, Xuzhou, China, ${ }^{3}$ Department of Rehabilitation Medicine, Ruijin Hospital, Shanghai Jiao Tong University School of Medicine, Shanghai, China, ${ }^{4}$ Department of Rehabilitation Medicine, Shanghai Jiao Tong University Affiliated Sixth People's Hospital, Shanghai, China, ${ }^{5}$ Department of Rehabilitation Medicine, Shanghai Shangti Orthopaedic Hospital, Shanghai, China

Neuropathic pain (NP) is poorly managed, and in-depth mechanisms of gene transcriptome alterations in NP pathogenesis are not yet fully understood. To determine microRNA-related molecular mechanisms of NP and their transcriptional regulation in NP, PubMed, Embase, Web of Science and CINAHL Complete (EBSCO) were searched from inception to April 2021. Commonly dysregulated miRNAs in NP were

Edited by:

Wenbin Guo,

The James Hutton Institute, United Kingdom

Reviewed by: Parisa Gazerani, Oslo Metropolitan University, Norway Hung-Chen Wang, Kaohsiung Chang Gung Memorial Hospital, Taiwan

*Correspondence: Pei-Jie Chen chenpeijie@sus.edu.cn

Xue-Qiang Wang wangxueqiang@sus.edu.cn

Specialty section: This article was submitted to RNA Networks and Biology, a section of the journal

Frontiers in Molecular Biosciences

Received: 21 September 2021 Accepted: 27 December 2021 Published: 13 January 2022

Citation:

Zheng $Y$ - L, Su X, Chen Y-M, Guo J-B,

Song $G$, Yang $Z$, Chen $P-J$ and Wang $X-Q$ (2022) microRNA-Based Network and Pathway Analysis for Neuropathic Pain in Rodent Models.

Front. Mol. Biosci. 8:780730

doi: $10.3389 /$ fmolb.2021.780730 assessed. The putative targets of these miRNAs were determined using TargetScan, Funrich, Cytoscape and String database. A total of 133 literatures containing miRNA profiles studies and experimentally verify studies were included. Venn analysis, target gene prediction analysis and functional enrichment analysis indicated several miRNAs (miR200b-3p, miR-96, miR-182, miR-183, miR-30b, miR-155 and miR-145) and their target genes involved in known relevant pathways for NP. Targets on transient receptor potential channels, voltage-gated sodium channels and voltage-gated calcium channels may be harnessed for pain relief. A further delineation of signal processing and modulation in neuronal ensembles is key to achieving therapeutic success in future studies.

Keywords: bioinformatics analysis, miRNA, neuropathic pain, functional enrichment analysis, biomarker

\section{INTRODUCTION}

Neuropathic pain (NP) is poorly managed and causes by a disease or lesion of the somatosensory nervous system (Cohen and Mao, 2014), accompanied by metabolic diseases, mechanical trauma or tumor invasion; it concerns various pathophysiological changes within the peripheral and/or central nervous system (Costigan et al., 2009; Zhang Y. H et al., 2021). A distinctive feature of peripheral neuropathic pain is mechanical allodynia, which is triggered by light touch (Costigan et al., 2009; Liu et al., 2018). A number of rodent NP models that have been established to shape exploration efforts in the pathophysiological mechanisms of NP in the nervous system include the sciatic chronic constriction injury (CCI), spared nerve injury (SNI), spinal nerve ligation (SNL), sciatic nerve transection, diabetic neuropathy and drug-induced neuropathy models. Although human perception of pain is subjective that it cannot be completely duplicated in animal models, tactile allodynia in rodent models is regarded to be a corresponding pattern for neuropathic mechanical hypersensitivity in patients. These models commonly provide a clear picture of cause-effect relationship or particular biomarkers in NP. 
In recent years, several studies have comprehensively discussed epigenetic modifications have dominated our understanding about activation and suppression of various gene expressions in the persistent and development of NP models (Penas and Navarro, 2018; Guo et al., 2019; An et al., 2021; Zheng et al., 2021). Unfortunately, in-depth mechanisms of gene transcriptome alterations in NP pathogenesis are not yet fully understood. Our previous study (Guo et al., 2019) have investigated the biological function of microRNAs (miRNAs) in frequently used NP rat model like CCI, SNI and SNL. Although miRNAs are noncoding RNAs containing only 19-25 nucleotides and are not directly involved in peptide synthesis, they markedly control biological processes by affecting mRNA stability, as well as protein translation (Lopez-Gonzalez et al., 2017; Song et al., 2020). Nervous tissues, such as dorsal root ganglia (DRG), spinal cord and prefrontal cortex (PFC), are of considerable concern in terms of the therapeutic potential of miRNAs in NP (Tang et al., 2021). A number of miRNAs, such as miR183 cluster, miR-155, miR-145, miR-203 and miR-200b/429, are detected in nervous tissues and are involved in NP by affecting neuronal excitability, neuroinflammation, or neuronal plasticity (Hamada et al., 2014; Peng et al., 2017; Shi et al., 2018; Zhang Y et al., 2020). In addition, the dysregulation of certain miRNAs mediates downstream molecular mediator of pain, which contain transient receptor potential receptor channels, purinergic receptors, and voltage-gated sodium and calcium channels (Yan et al., 2020a; Yeh et al., 2020; Li et al., 2021). Although numerous studies have attempted to provide novel mechanistic insights into the role of miRNAs roles in NP, the entire pain pathway still remains to be elucidated. Hence, we performed comprehensive literature search on the microRNA-related molecular mechanisms of NP, and bioinformatics analysis of their transcriptional regulation. This study may shed light on the enigmatic pathophysiology of NP.

\section{METHODS}

\section{Search Strategy}

A systematic search was performed in April 2021 on four electronic databases, namely, PubMed, Embase, Web of Science and CINAHL Complete (EBSCO). The terms used for search words included the following: "neuropathic pain", "Neuralgia", "Nerve pain", "sciatica", "chronic constriction injury", "spinal nerve ligation", "spared nerve injury", "chronic compression dorsal root ganglion", "CCI", "SNI", "SNL" or "CCD") and ("microRNA", "micro-RNA", "mir", or "miRNA"). No language restrictions were employed. The reference lists of all identified articles were examined. Full details of the search strategy for all databases can be found in Supplementary Appendix S1. Subsequently, all search results were imported in EndNote X7 (Thomson Research Soft, Stamford, United States). Duplicate items, reviews, abstracts and full texts were removed.

\section{Inclusion Criteria}

Compared with our previous study (Guo et al., 2019), this study focused not only on NP rat model like CCI, SNI and SNL, but also on mouse model, meanwhile, contained drugand disease-induced neuropathy models. So, several unambiguous inclusion criteria were listed as follows: 1) types of studies: only original articles exploring miRNAs' roles in NP through comparison with the rodent models in NP condition and those without NP. Conference abstracts, conference presentations, book chapters, book reviews, case studies, meta-analysis, news items and corrections were excluded; 2) types of animal models: a. mouse or rat models of NP surgical models containing CCI, SNI, SNL, sciatic nerve transection, partial sciatic nerve injury, brachial plexus avulsion and trigeminal neuralgia, b. mouse or rat models of drug-induced NP models, including vincristine, cisplatin, oxaliplatin, bortezomib and taxanes, c. mouse or rat models of disease-induced NP models, including diabetesinduced neuropathy, post-herpetic neuralgia and cancer pain; 3) types of samples: nervous tissues, such as brain, spinal cord, DRG, sciatic nerve, and nervous cells, just like microglia, astrocytes and DRG neurons; and 4) types of measurements: miRNA expression measured in the way of polymerase chain reaction, microarray analysis or TaqMan low density array.

\section{Data Extraction}

Data were extracted independently by two authors ( $\mathrm{Su} \mathrm{X}$ and Chen YM). For each included study, the first author's family name, publication year, country, experimental design (e.g., experimental models, region used) and miRNA details (e.g., expression alteration, target genes and functions) were extracted. In case of disagreement, the two authors discussed the issue or consulted a third author (Wang XQ).

\section{Bioinformatics Analysis of miRNAs and Its Targets}

Venn diagram analysis showed the number of overlapping genes by Functional Enrichment analysis tool (FunRich v3.1.3; installation package downloaded from http://www. funrich.org/). The fold value for dysregulated miRNAs in the matrix table is greater than or equal to 1.5. TargetScan (http://www.targetscan.org/) was used to predict the target genes of miRNAs and further verified the functional specificity of miRNAs. Then, protein-protein interaction (PPI) networks were established to having an integral understanding about the association among the overlapping targets of differentially expressed miRNAs. Additionally, PPI data were achieved from the String database (http://string-db.org/) and imported in Cytoscape v3.7.2 (Shannon et al., 2003) to draw a map. The high confidence score with $>0.7$ was defined to establish PPI network. Gene Ontology (GO) annotation was used to probe the functional roles of putative targets from three aspects, namely, biological process (BP), cellular component (CC) and molecular functions (MF). Kyoto 
6653 records identified through database searching

125 form PubMed 1270 from EMBASE

3115 from CINAHL Complete (EBSCO) 1328 from Web of science

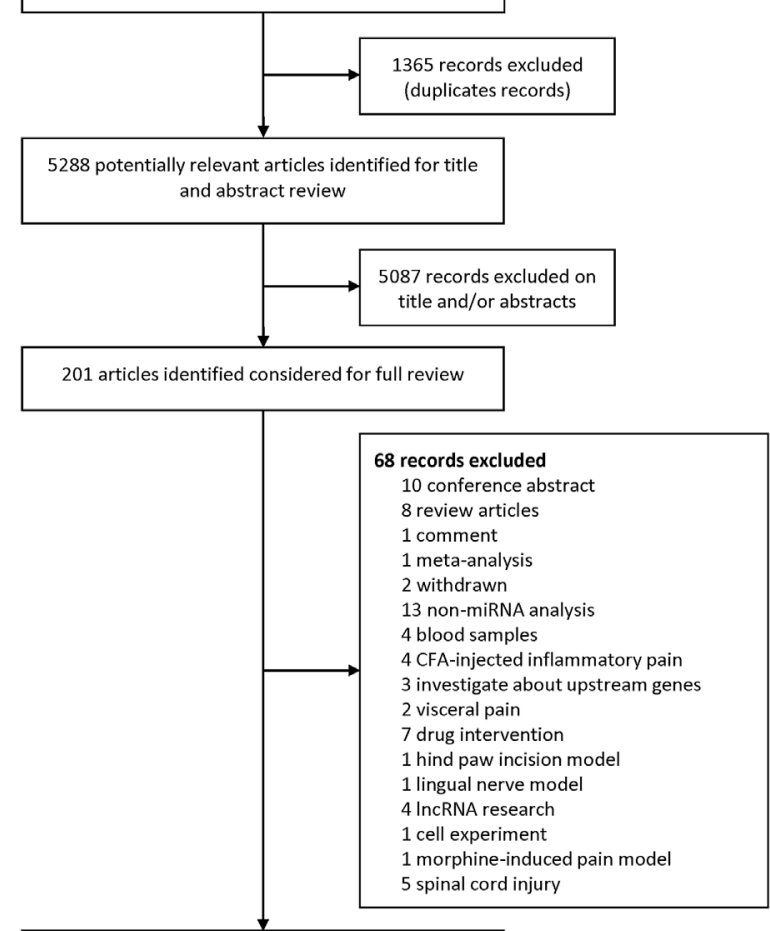

133 articles included in synthesis

FIGURE 1 | Flow chart of the study selection procedure.

Encyclopedia of Genes and Genomes (KEGG) analysis was conducted to predict the metabolic pathways of gene products. GO analysis and KEGG analysis were performed in DAVID (The Database for Annotation, Visualization and Integrated Discovery; https://david.ncifcrf.gov/). Significantly targeted pathways were identified by Fisher exact test with $p$-value <0.05. GraphPad Prism 8.0.1 (GraphPad Software, La Jolla California United States, http://www.graphpad.com) was used to present the enrichment results.

\section{RESULTS}

We identified 6,653 records from online databases. After duplicate records were removed, 5,288 items remained. Two authors assessed the title and abstract and found that 201 articles were pushed on full-text review. At last, 133 literatures met the eligibility criteria and were included (excluded articles are listed in Supplementary Table S1). Among these included studies, 23 were about miRNA profiles (Supplementary Tables S2, S3), 113 were about miRNA experimental verification and three (Dong et al., 2014; Lu et al., 2017; Liu L et al., 2020) were both about miRNA profile and experimental verification. Among the 113 miRNA experimental verification articles, 96 are about NP surgical models (Supplementary Table S4), 6 are drug-induced NP models (Supplementary Table S5), and 11 are disease-induced NP models (Supplementary Table S6). The flowchart of the study selection procedure is shown in Figure 1.

\section{Study Characteristics}

Details on the characteristics of articles that met inclusion criteria are shown in Supplementary Tables S2-S6. A total of 23 articles examined differential expression of miRNAs in NP rodent model's expression profiling study (Supplementary Tables S2, S3). Among these articles, the quantity of the significantly dysregulated miRNAs ranged from 1 to 59. As of April 2021, there are no expression profiles papers on drug induced NP models, 21 expression profiles papers on NP surgical models and 2 expression profiles papers on drug induced NP models. $34.8 \%$ $(8 / 23)$ of the studies collected DRGs and $43.5 \%(10 / 23)$ collected $\mathrm{SDH}$ to examine differentially expressed miRNAs between NP rats/mice and sham group rats/mice. In the latest study (Chen et al., 2021), miRNA changes in DRG after SNI were analyzed by DESeq2 and verified by qRT-PCR. Three miRNAs (miR-351-5p, miR-125a-5p and miR-125b-5p) were significantly downregulated, whilst no up-regulated miRNA was mentioned.

A total of 113 articles experimentally verified that 91 miRNAs might take part in NP regulation (Supplementary Tables S4-S6). Specifically, 96 articles verified 79 miRNAs in NP surgical models (Supplementary Table S4), 6 articles verified 5 miRNAs in druginduced NP models (Supplementary Table S5) and 11 articles verified 11 miRNAs in disease-induced NP models (Supplementary Table S6). The function of these NP-related miRNAs was generally categorized by neuroinflammation, neuronal adaptivity, neuronal excitability, neuronal plasticity, neuronal proliferation, DNA methylation, neuroimmune and GABAergic synapses excitability.

Supplementary Table S4 shows a comparison of the shamoperated group and five NP surgical models, namely, SNL, SNI, CCI, sciatic nerve transection, partial sciatic nerve ligation and CFA-induced prosopalgia. As for tissue analysis, miRNA expression was altered in the nervous tissues and nervous cells. Nervous tissues included DRG, spinal cord, trigeminal ganglions, caudal medulla and sciatic nerve; nervous cells included microglia, DRG neurons and astrocytes. Briefly, $61.5 \%(59 / 96)$ of experimental studies evaluated the miRNA expression in spinal cord, 38.4\% (37/96) in DRG, 4.1\% (4/96) in sciatic nerve, $2.1 \%(2 / 96)$ in trigeminal ganglions, $1.0 \%(1 / 96)$ in nucleus accumbens, $1.0 \%(1 / 96)$ in caudal medulla and $1.0 \%$ $(1 / 96)$ in sural nerve. Moreover, the expression level of several miRNAs (Jiang et al., 2016; Tramullas et al., 2018; Wang et al., 2018; Zhang X et al., 2020; Zhou et al., 2020; Sun et al., 2021; Zhang J et al., 2021) in DRG and the spinal cord were changed. In terms of included studies, 58 miRNAs were down-regulated in the NP process, of which miR-98, miR-96, miR-182, miR-183, miR7a, miR-30b, miR-206, miR-200b, miR-429, miR-150 and miR145 were reported two times or more. 26 miRNAs were upregulated, of which miR-21, miR-155 and miR-195 were reported two times or more. In the down-regulated and up-regulated 


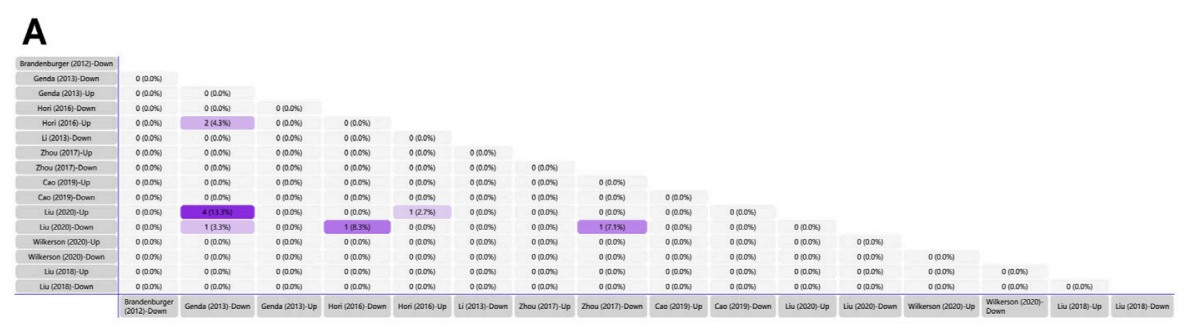

B

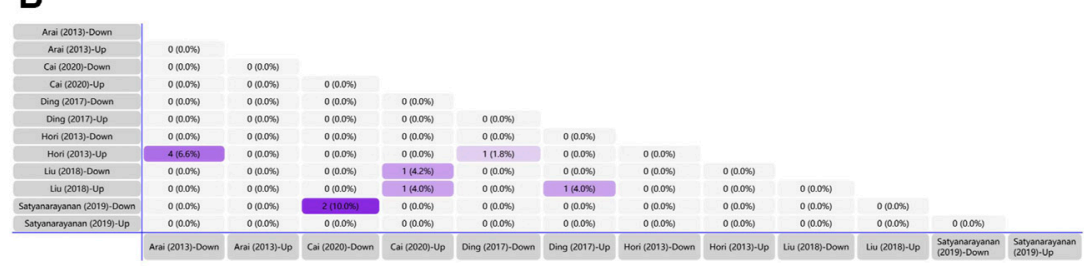

FIGURE 2 | Tabular Venn diagram analysis. (A) Matrix table analysis for miRNAs expression profiles in spinal cord of NP surgical models. The number and percentage of co-regulated miRNAs were highlighted. Overlapping miRNAs: 2 (4.3\%): miR-21, miR-27b; 4 (13.3\%): miR-22, miR-377, miR-7a, miR-21; 1 (3.3\%): miR365; 1 (8.3\%): miR-214; 1 (2.7\%): miR-21; 1 (7.1\%): miR-184. (B) Matrix table analysis for miRNAs expression profiles in brain of NP surgical models. The number and percentage of co-regulated miRNAs were highlighted. Overlapping miRNAs: 4 (6.6\%): miR-132, miR-151-3p, miR-186, miR-204; 2 (10.0\%): miR-200b-3p, miR182; 1 (4.2\%): miR-370-5p; 1 (4.0\%) left: miR-873-5p; 1 (4.0\%) right: miR-205; 1 (1.8\%): miR-539.

miRNAs, there were five overlapping genes, namely, miR-23a, miR-142-3p, miR-21-5p, miR-15a and miR-101. Among them, miR-23a expression decreased in SDH (Pan et al., 2018) and increased in DRGs (Zhang Y et al., 2021); miR-142-3p expression decreased in DRGs (Zhang et al., 2018) and increases in the sciatic nerve (Li et al., 2021); miR-21-5p expression decreased in the dorsal spinal cords (Zhong et al., 2019) and increased in DRGs (Simeoli et al., 2017); miR-15a and miR-101 showed contradictory results in the spinal cords and $\mathrm{SDH}$, respectively. Cai et al. (2020) indicated miR-15a was downregulated in spinal cords, while $\mathrm{Li} \mathrm{T}$ et al. (2019) indicated miR-15a was up-regulated. Xie et al. (2020) revealed miR-101 was down-regulated in SDH, whilst Qiu et al. (2020) disclosed miR-101 was up-regulated.

Six studies verified that five miRNAs changed in NP induced by chemotherapeutic drugs, such as paclitaxel, oxaliplatin and bortezomib (Supplementary Table S5). $\mathrm{SDH}$ and DRGs were collected for measurement. miR-30b5p (Li L et al., 2019) and miR-141-5p (Zhang and Chen, 2021) were down-regulated in chemotherapeutic drug-induced NP; miR-500 (Huang et al., 2016), miR-15b (Ito et al., 2017) and miR-155 (Miao et al., 2019; Duan et al., 2020) were upregulated. NP induced by cancer pain and diabetes are shown in Supplementary Table S6. Four studies showed that three miRNAs were down-regulated (Elramah et al., 2017; Wu X. P. et al., 2019; Liu C et al., 2020) (miR-124, miR-329 and miR-300), and 1 miRNA was up-regulated (Gandla et al., 2017) (miR-34c-5p) in NP induced by bone cancer pain; and 7 studies indicated 5 down-regulated miRNAs (Yang et al., 2017; Feng et al., 2018; Wu B. et al., 2019; Yan et al., 2020b; Wu et al., 2020) (miR-190a-5p, miR146a, miR-193a, miR-145 and miR-590-3p) and 2 upregulated miRNAs (Chen et al., 2019; Chang et al., 2020) (miR-155 and miR-133a-3p) in diabetic neuropathic pain.



FIGURE 3 | Matrix table analysis for miRNAs expression profiles of disease-induced NP models. The number and percentage of co-regulated miRNAs were highlighted. The two overlapping miRNAs are miR-466i-3p and miR-466g.

\section{Target Prediction and Venn Diagram Analysis}

We generated matrix tables catalogized by DRG, spinal cords and brain tissues using FunRich (http://www.funrich.org), and utilized array data from Supplementary Table S2. It reveals the percentage and quantity of corporately expressed miRNAs in the expression profiling studies. In the matrix table for spinal cords of surgical NP rodents (Figure 2A), miR-365, miR-214 and miR-184 were down-regulated in two or more studies, and miR21 was up-regulated in two studies. As for brain tissues (Figure 2B), miR-200b-3p and miR-182 were down-regulated in two studies, miR-873-5p was up-regulated, whereas none commonly down-regulated nor up-regulated miRNA in DRGs was found (Supplementary Figure S1). According to array data 


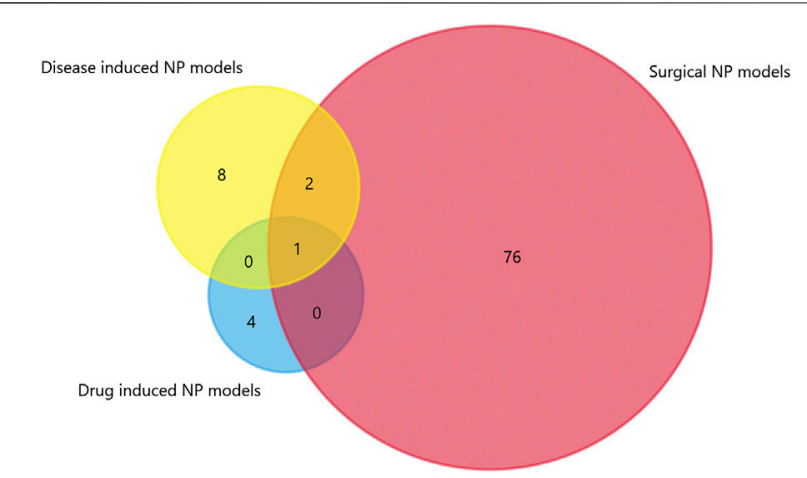

FIGURE 4 | The intersection of experimentally verified miRNAs in three types of rodent NP models. Overlapping miRNAs: 2: miR-34c-5p, miR-145; 1 : $\mathrm{miR}-155$.

from Supplementary Table S3, Venn diagram was drawn using FunRich. It shows that miR-466i-3p and miR-466g were commonly down-regulated (Figure 3). Experimentally verified studies were analyzed, and rodent NP models were divided into surgical, disease-induced and drug-induced NP models. The Venn diagram shows that miR-155 was the only miRNA that was verified in all three types of NP models with up-regulated expression; miR-34c-5p was commonly up-regulated and miR145 was commonly down-regulated in disease-induced and druginduced NP models (Figure 4).

TargetScan was used to predict the miRNAs' target genes and then FunRich was used to draw Venn diagrams. There were 225 overlapping target genes in the two down-regulated miRNAs (miR-200b-3p and miR-182), which detected from the cerebral cortex; but none in the three down-regulated miRNAs (miR-365, miR-214 and miR-184), which detected from spinal cords (Figure 5A and Supplementary Figure S2). Analysis of experimentally verified studies of surgical NP indicated that miR-96, miR-182, miR-183, miR-30b and miR-145 were down-regulated both in spinal cords and DRGs. Furthermore, these five miRNAs were reported in at least two experimental studies. The miR-183 cluster comprised miR-96, miR-182 and miR-183. This cluster shared the same sequence homology, so it is reasonable to merge the target genes of miR-96, miR-182 and miR-183 for Venn diagrams analysis. A total of 63 overlapping target genes were present in the miR-183 cluster, miR-30b and miR-145 (Figure 5B). Moreover, 13 overlapping target genes were found in three repeatedly verified up-regulated miRNAs (miR-21, miR-155 and miR-195) (Supplementary Figure S3).

\section{PPI Network Analysis}

The PPI data of overlapping genes in pain-related nervous tissues were obtained from STRING database, and the interaction networks were constructed using Cytoscape software (v3.7.2). The results of PPI analysis are shown in Figure 6 and Supplementary Figure S4. The results showed 94 genes exhibiting interactions in 225 overlapping target genes of miR200b-3p and miR-182 (Figure 6). A total of 17 genes showed interactions in 63 overlapping target genes of miR-183 cluster,
miR-30b and miR-145 (Supplementary Figure S4). Each edge in the PPI network represented protein-protein associations. Large sizes and dark colors of edges meant high value of combined scores, otherwise, small sizes and bright colors of edges meant low value of combined scores. As shown in Figure 6, muscle and microspikes RAS (Mras) connected with phosphatidylinositol 3kinase, catalytic, alpha polypeptide (Pik3ca), and cAMP responsive element binding protein 1 (Creb1) connected with E1A Binding Protein P300 (EP300) displayed highest connective score with combined score of 0.997 and 0.996 , respectively. In addition, combined score of 0.998 was the highest score in Supplementary Figure S4, which is insulin like Growth factor 1 receptor (Igf1r) connected with insulin receptor substrate 1 (Irs1).

Furthermore, the experimentally verified miRNAs in Supplementary Table $\mathbf{S 4}$ and their corresponding target genes were also mapped (Figure 7). Four down-regulated miRNAs (miR-96, miR-183, miR-384 and miR-30b) directly targeted Nav1.3. Similarly, four up-regulated miRNAs (miR-19a, miR-221, miR-155 and miR-665) and one down-regulated

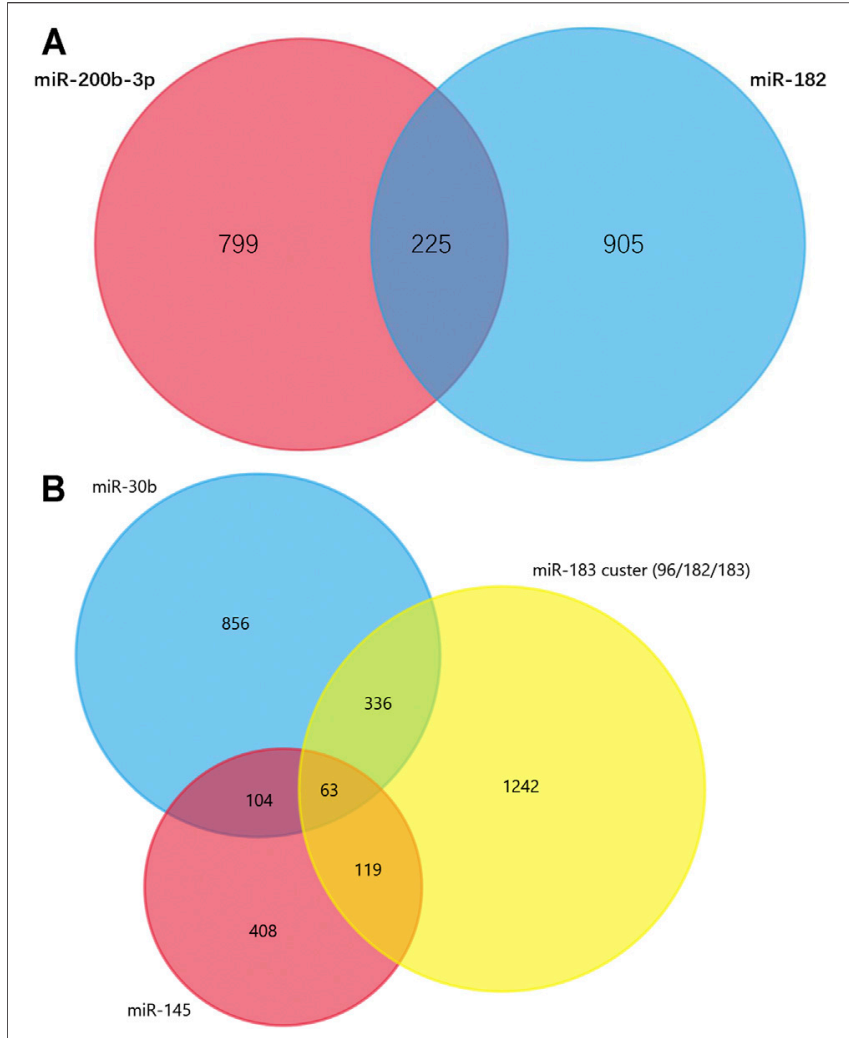

FIGURE 5 | Venn diagram analysis. (A) Overlapping target genes of miR182 and miR-200b in cerebral cortex. These down-regulated genes were showed in Supplementary Table $\mathbf{S} \mathbf{2}$ and have been observed in more than one studies. (B) Overlapping target genes of miR-96, miR-182, miR183, miR-145 and miR-30b in dorsal root ganglions. These five downregulated genes were showed in Supplementary Table $\mathbf{S} \mathbf{4}$ and have been observed in two or more experimentally verified studies. miR-96, miR-182, miR-183 formed miR-183 cluster, so their target genes were combined. 


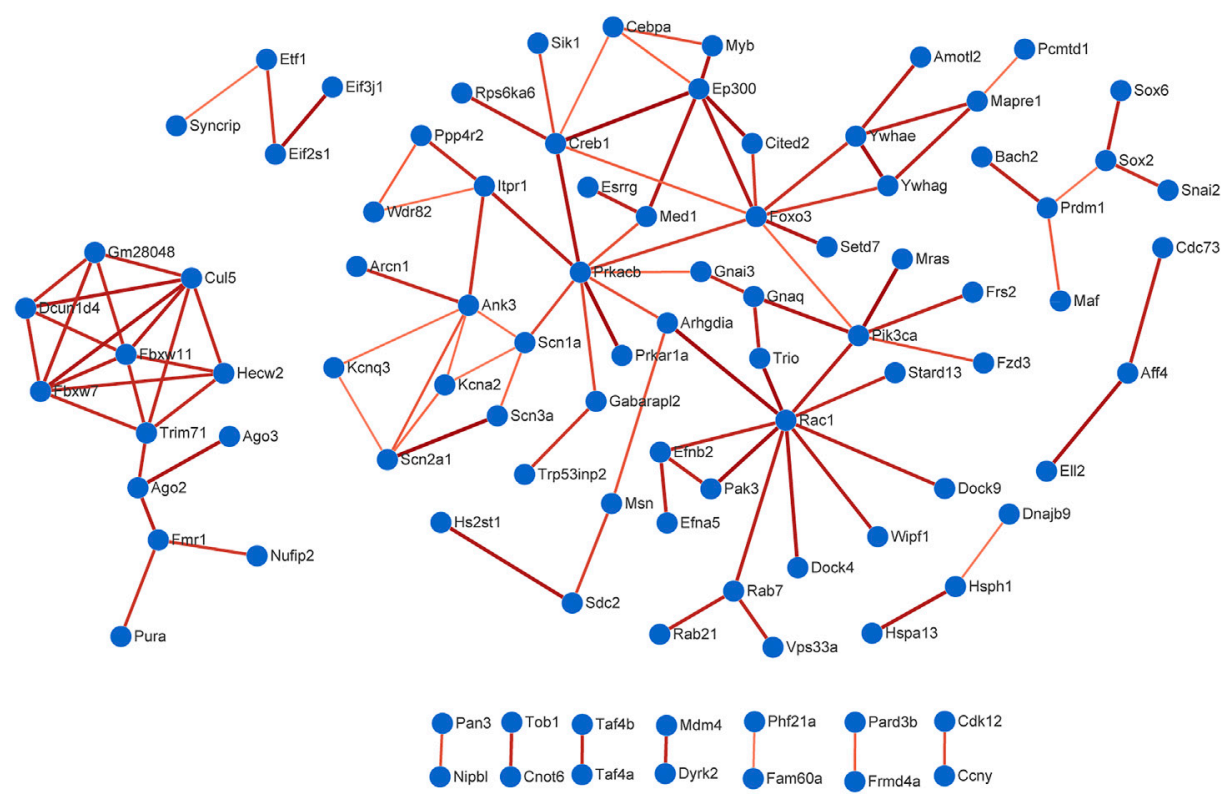

FIGURE 6 | Protein-protein interaction (PPI) analysis. A total of 94 genes exhibited interactions in 225 overlapping target genes of miR-200b-3p and miR-182. Large sizes and dark colors of edges meant high value of combined scores. High confidence score of 0.7 was selected to construction PPI network.

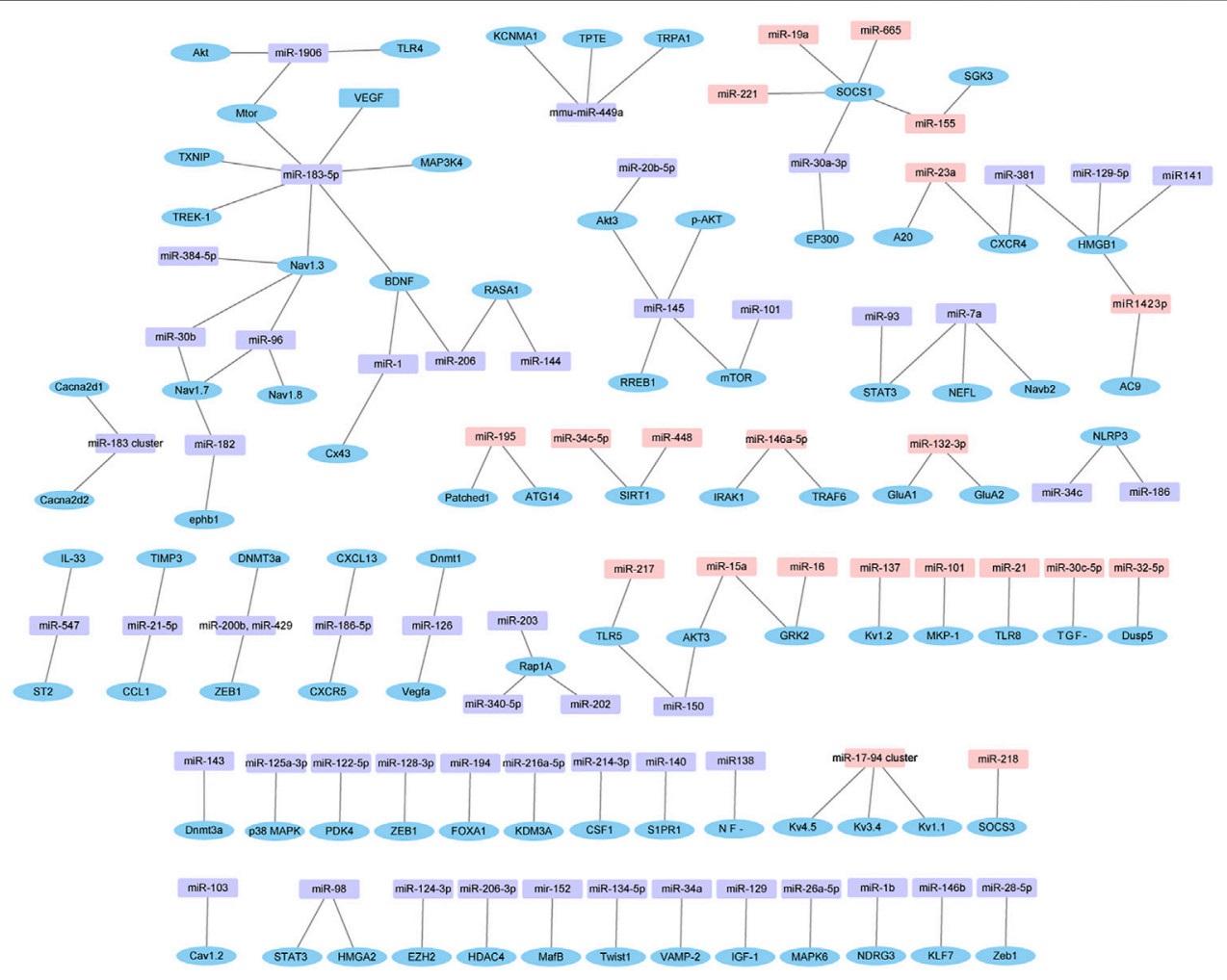

FIGURE 7 |Dysregulated miRNAs-target gene network. The network is based on dysregulated miRNAs and their target genes identified in Supplementary Table S4. Pink in rectangle represent up-regulated miRNAs, purple in rectangle represent down-regulated miRNAs, and blue in ellipse represent target genes. 


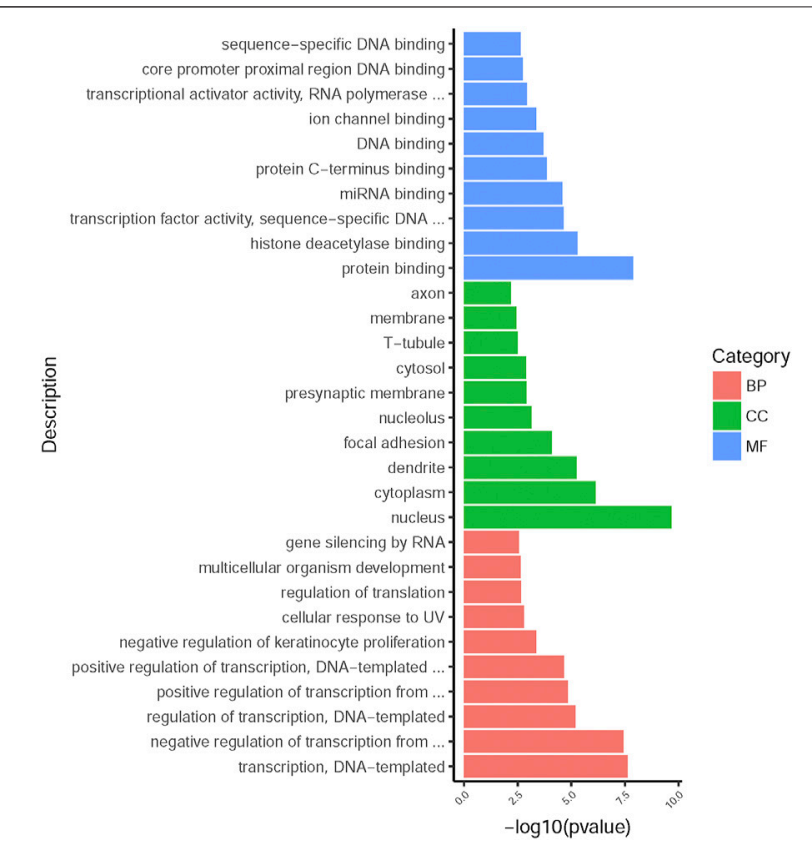

FIGURE 8 | GO annotation enrichment analysis. The top 10 high enrichment score terms in biological process, cellular components and molecular functions. GO: gene ontology; BP: biological process; CC: cellular components; MF: molecular functions.

miRNA (miR-30a-3p) directly targeted suppressor of cytokine signaling 1 (SOCS1).

\section{Functional Enrichment Analysis}

225 overlapping target genes of miR-200b-3p and miR-182 were analyzed. The results of GO analysis consisted of cellular components (e.g., nucleus, GO: 0005634), molecular functions (e.g., protein binding, GO: 0005515), and biological process (e.g., transcription, DNA-templated, GO: 0006351). Overall, 133 GO terms showed significant enrichment $(p<0.05)$, and the top 10 terms with enrichment score in each aspect are presented in Figure 8. A number of enriched terms relevant to the nervous system included dendrite (GO: 0005634), synapse (GO: 0045202), axon (GO: 0030424), nervous system development (GO: 0007399) and neuron projection (GO: 0043005). Furthermore, KEGG analysis showed 26 pathways showed significant enrichment $(p<0.05)$, and the top 20 pathways with enrichment score are presented in Figure 9. Many of the enriched pathways were associated with the nervous system, including cholinergic synapse, neurotrophin signaling pathway, dopaminergic synapse, hippo signaling pathway and glutamatergic synapse.

\section{DISCUSSION}

In this study, miRNAs in nervous tissues of NP rodent models were comprehensively analyzed using 23 miRNA profile articles and 113 miRNA experimental verification articles. Compared with the sham-operated group, a total of 91 miRNAs verified by 113 experimental articles were differentially expressed in the NP group. The potential functional specificity of the differentially expressed miRNAs for pain was determined by GO and KEGG pathway analysis. The results suggest that miRNAs served a vital role in NP development and potentially novel strategies for NP management.

NP pathogenesis is complex and remains poorly understood. Regions of DRGs, SDH and anterior cingulate cortex (ACC) form the somatosensory pathway from primary sensory neurons to afferent nerve to central nervous system (CNS) in NP (Guo et al., 2019). DRGs contain most of the body's sensory neurons, and transmitted sensory messages from receptors, such as thermoreceptors and nociceptors, are active participants in the signalling process (Pope et al., 2013). SDH is the relay station of nociceptive stimuli to process somatosensory information and is characterized as an unambiguous laminar structure with a quantity of excitatory and inhibitory interneurons (Peirs and Seal, 2016). ACC is a dominant cortical area implicated in diverse neurological processes, such as nociception, cognition and emotion and plays a critical role in emotional/aversive component of pain (Tsuda et al., 2017).

Numerous circuits and molecules were identified as potential biomarkers and regulators in NP. The renowned mediators contain the transient receptor potential (TRP) channels (Lu et al., 2017; Miao et al., 2019; Duan et al., 2020; Zhang and Chen, 2021), voltage-gated sodium channels ( $\left.\mathrm{Na}_{\mathrm{V}} \mathrm{s}\right)$ (Chen et al., 2014; Lin et al., 2014; Shao et al., 2016; Su et al., 2017; Cai et al., 2018; Li L et al., 2019; Yang et al., 2019; Yan et al., 2020b; Ye et al., 2020; Sun et al., 2021), and voltage-gated calcium channels (CaVs) (Favereaux et al., 2011; Gandla et al., 2017; Peng et al., 2017). In DRG and SDH, TRP channels act as transducers for

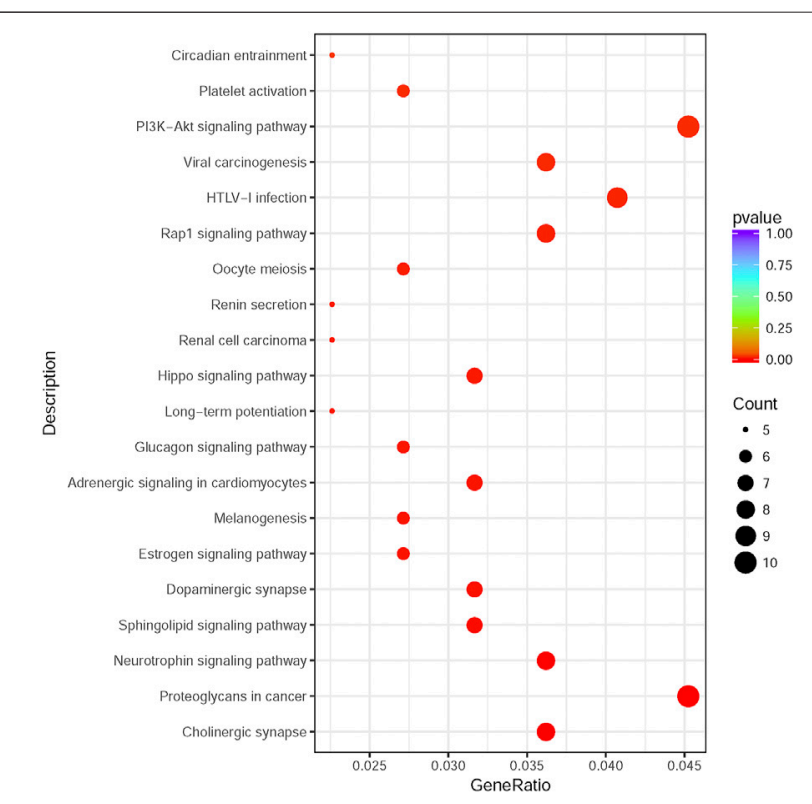

FIGURE 9 |KEGG pathway enrichment analysis. The top 20 enrichment score pathways were showed. KEGG: Kyoto Encyclopedia of Genes and Genomes. 
selectively activating sensory neurons and conveying diverse kinds of stimuli involving chemical, mechanical, and thermal stimuli. TRP ankyrin 1 (TRPA1) is one of branches of TRP channels contributing to NP. In NP surgical models and druginduced NP models, TRPA1 is overexpressed and targeted by miR-155 (Miao et al., 2019; Duan et al., 2020), miR-141-5p (Zhang and Chen, 2021), mmu-miR-449a (Lu et al., 2017). Ectopic action potential generation is considered to be one of causes of NP formulation. $\mathrm{Na}_{\mathrm{V}} \mathrm{s}$ intrinsically contribute to ectopic activity generation (Bannister et al., 2020) and are typically encoded by different subunits, $\mathrm{Na}_{\mathrm{V}} 1.1-\mathrm{Na}_{\mathrm{V}} 1.9$. DRG neurons contain the most varied kind of $\mathrm{Na}_{\mathrm{V}}$ subtypes. Among them, $\mathrm{Na}_{V} 1.3$ (Chen et al., 2014; Ye et al., 2020), $\mathrm{Na}_{V} 1.6$ (Li L et al., 2019), $\mathrm{Na}_{\mathrm{V}} 1.7$ (Sun et al., 2021) and $\mathrm{Na}_{\mathrm{V}} 1.8$ (Yan et al., 2020b) are overexpressed in NP and are targeted by miR-96, miR-183, miR182 and miR-30b. In addition, Sun and colleagues (Sun et al., 2021) demonstrated that miR-96 knockout mice showed thermal and mechanical allodynia. This allodynia was alleviated by intraperitoneal or intrathecal injection of $\mathrm{Na}_{\mathrm{V}} 1.7$ or $\mathrm{Na}_{\mathrm{V}} 1.8$ blockers. SNI-induced suppressed expression of miR-96 in $\mathrm{SDH}$ showed negative correlation to overexpression of $\mathrm{Na}_{\mathrm{V}} 1.7$ $\mathrm{Na}_{\mathrm{V}}$ 1.9. This dysregulation promotes the NP process, and can be attenuated by intrathecal injection of corresponding $\mathrm{Na}_{V} \mathrm{~s}$ blockers. Thus, alternation in the expression of $\mathrm{Na}_{\mathrm{V}} \mathrm{s}$ in nerve injury-induced NP might be efficacious for pain relief. Furthermore, increased expression and function of $\mathrm{Ca}_{V} \mathrm{~s}$ also caused NP by increasing transmitter release (Davies et al., 2007). $\mathrm{Ca}_{V} \mathrm{~s}$ are grouped into two classes, namely, high-voltage activated type and low-voltage activated type (also known as T-type) (Catterall et al., 2005). Apart from T-type $\mathrm{Ca}_{V} s, \mathrm{Ca}_{V} \mathrm{~s}$ have three types of subunits, namely, the al channel-forming subunit, the intracellular $\beta$ subunit and the $a 2 \delta$ auxiliary subunit. The $\alpha 2 \delta$ auxiliary subunit is composed of two disulfide-bonded polypeptides ( $\alpha 2$ and $\delta$ ) (Dolphin, 2018). Furthermore, $\mathrm{Ca}_{\mathrm{V}} 1.2$ that encodes the al subunit of $\mathrm{Ca}_{\mathrm{V}} \mathrm{s}$ targeted by miR-103 is upregulated in SNL rats (Favereaux et al., 2011); $\mathrm{Ca}_{\mathrm{V}} 2.3$, which targeted by miR-34c-5p, is downregulated in bone metastatic pain mice (Gandla et al., 2017). Both mRNA and protein levels of the $\alpha 2 \delta-1$ subunit and $\alpha 2 \delta-2$ subunit of $\mathrm{Ca}_{\mathrm{V}}$ are upregulated in SNI mice and are correlated with mechanical allodynia (Peng et al., 2017). The miR-183 cluster (miR-183/96/182) controls over $80 \%$ of NPrelated genes and scales mechanical allodynia by modulating the $a 2 \delta-1$ subunit and $\alpha 2 \delta$-2 subunit (Peng et al., 2017). Thus, postinjury alteration in $a 2 \delta$ expression level, bound with upstream miRNAs dysregulations, provides convincing evidence for potential biomarkers and regulators in NP and therapeutic possibilities of $\mathrm{Ca}_{\mathrm{V}} \mathrm{s}$.

Several studies indicated that epigenetic changes, translational modification and post-translational control influence NP development and management. Epigenetic changes are considered to involve histone acetylation and DNA methylation. As such, gene expression is regulated but does not change the coding sequence. DNA methylation likely reduces transcription efficiency, whilst histone acetylation in DNA presents the active transcriptional region (Jenuwein and Allis, 2001). Methyl-CpG-binding protein 2 (MeCP2), a protein with affinity for methylated DNA and repressing transcription from methylated gene promoter, is vital for neuronal proliferation and embryonic development. Manners et al. (Manners et al., 2016) found that SNI caused the redistribution of $\mathrm{MeCP} 2$ to methyl-CpG binding domain. Enriched $\mathrm{MeCP} 2$ can bind to the miR126 locus in NP condition and restrain miR-126 transcription. Repressed miR-126 expression contributes to the up-regulation of its two target genes Dnmt1 and Vegfa in SNI mice. DNA methyltransferase 3A (Dnmt3a) was increased in injured DRG. $\mathrm{Xu}$ et al. (Xu et al., 2017) indicated that SNL-induced miR-143 downregulation is a negative regulator in Dnmt3a expression in DRG. Furthermore, histone deacetylases (HDACs) were implied in the mechanisms of transcriptional regulation, cell cycle progression, neuron degeneration and regulation of neuronal plasticity. Overexpression of HDAC4 alleviated the effects of miR-206-3p on NP (Wen et al., 2019). Overall, miRNAregulated DNA methylation and histone acetylation may be a potential target for NP therapeutic management. The interactions between transcriptional factors (TFs) and mitogen-activated protein kinases (MAPKs) are also involved in NP. MAPK6 up-regulation in SDH of CCI rats was deemed to be a direct downstream target gene of miR26a-5p (Zhu et al., 2018). Suppressed miR-125a-3p contributed to p38 MAPK up-regulation in rat trigeminal ganglions at different time points with prosopalgia (Dong et al., 2014). Inhibition of miR-155 reduces NP during chemotherapeutic bortezomib via downstream signals p38MAPK (Duan et al., 2020). Additionally, in diabetes mellitus rats, miR-133a-3p antagomir administration lightened diabetic NP and down-regulated p38 MAPK (p-p38) phosphorylation (Chang et al., 2020). Hence, pain-related miRNAs and proteins may reveal critical insights into how neurons process incoming stimuli inputs and attempt to create an effective method for low risk of inflammation and NP management.

PPI network analysis may help predicted some target genes to broaden the treatment options for NP in the near future. Mras connected with Pik3ca displayed highest connective score with combined score of 0.997 predicted by PPI network. Mras is a member of the Ras family of small GTPases that impacts mouse embryonic stem cell plasticity and neurogenesis (Mathieu et al., 2013). Pik3ca is usually described as an oncogenic gene and related to PI3K-AKT signaling pathway (Koundouros et al., 2020), which is in line with Figure 9. Another pair with high combined score of 0.996 is Creb1 and EP300. Creb1 encodes a transcription factor. This protein activity is regulated by protein kinase A (Maekawa et al., 2008) and also related to PI3K-AKT signaling pathway (Rodon et al., 2014). Creb1 was recognized as one of most enriched genes in brain and found to regulate a quite number of downstream genes in NP process (Yan et al., 2019). EP300 is identified as a transcriptional co-activator and is important in the processes of cell proliferation and differentiation (Son et al., 2019). Knockdown of miR-30a-3p in L4-6 spinal dorsal toot increased EP300 level and induced NP (Tan et al., 2020). At present, there is no study targeting Mras 
and Pik3ca in neuropathic pain. Using Pik3ca inhibitors in NP model may be an initial attempt to ameliorate pain in the near future.

Bioinformatics insights into the potential neurobiological mechanisms correlative of NP were investigated in a comprehensive and systematic literature search framework. This framework provided guidance for literature validation of microRNA regulatory networks inferred from real experimental data. An extensive range of miRNAs was obtained to explore the relationship among their downstream genes via network and pathway-based analysis. Based on this analysis, it is helpful to form integrative research strategies from NP mechanisms to treatment. Some limitations still exist in this study. Firstly, a few microRNAs revealed contradictory results in NP process, which may be caused by differences in tissue collection timing, modeling methods and detected tissue region. Secondly, the GRADE system to evaluate level of evidence and Funnel plots for publication assessment are lacking. Although the outcomes deduced from bioinformatic analysis, to some extent, may exist heterogeneity from different study designs, network analysis combined pathway analysis can be more robust for possible false positive results.

In summary, we investigated miRNA expression and their target gene roles in NP. Bioinformatics analysis elucidates that several miRNAs (miR-200b-3p, miR-96, miR-182, miR-183, miR-30b, miR-155 and miR-145) and its target genes are involved in known relevant pathways of NP. Targets on voltage-gated sodium channels may be harnessed for pain relief. As knowledge of molecular, genetic and epigenetic mechanisms about pain specificity and neuron plasticity accrues from rodent models, further delineation of signal processing and modulation in neuronal ensembles is key to achieving therapeutic success in future studies.

\section{REFERENCES}

An, Q., Sun, C., Li, R., Chen, S., Gu, X., An, S., et al. (2021). Calcitonin GeneRelated Peptide Regulates Spinal Microglial Activation through the Histone H3 Lysine 27 Trimethylation via Enhancer of Zeste Homolog-2 in Rats with Neuropathic Pain. J. Neuroinflammation 18, 117. doi:10.1186/s12974-02102168-1

Bannister, K., Sachau, J., Baron, R., and Dickenson, A. H. (2020). Neuropathic Pain: Mechanism-Based Therapeutics. Annu. Rev. Pharmacol. Toxicol. 60, 257-274. doi:10.1146/annurev-pharmtox-010818-021524

Cai, L., Liu, X., Guo, Q., Huang, Q., Zhang, Q., and Cao, Z. (2020). MiR-15a Attenuates Peripheral Nerve Injury-Induced Neuropathic Pain by Targeting AKT3 to Regulate Autophagy. Genes Genom 42, 77-85. doi:10.1007/s13258019-00881-Z

Cai, W., Zhao, Q., Shao, J., Zhang, J., Li, L., Ren, X., et al. (2018). MicroRNA-182 Alleviates Neuropathic Pain by Regulating Nav1.7 Following Spared Nerve Injury in Rats. Sci. Rep. 8, 16750. doi:10.1038/s41598-018-34755-3

Catterall, W. A., Perez-Reyes, E., Snutch, T. P., and Striessnig, J. (2005). International Union of Pharmacology. XLVIII. Nomenclature and StructureFunction Relationships of Voltage-Gated Calcium Channels. Pharmacol. Rev. 57, 411-425. doi:10.1124/pr.57.4.5

Chang, L.-L., Wang, H.-C., Tseng, K.-Y., Su, M.-P., Wang, J.-Y., Chuang, Y.-T., et al. (2020). Upregulation of miR-133a-3p in the Sciatic Nerve Contributes to Neuropathic Pain Development. Mol. Neurobiol. 57, 3931-3942. doi:10.1007/ s12035-020-01999-y

\section{DATA AVAILABILITY STATEMENT}

The original contributions presented in the study are included in the article/Supplementary Material, further inquiries can be directed to the corresponding authors.

\section{AUTHOR CONTRIBUTIONS}

Conceptualization and Methodology, X-QW and P-JC; Investigation, $\mathrm{XS}, \mathrm{Y}-\mathrm{MC}$ and $\mathrm{J}-\mathrm{BG}$; Formal analysis: $\mathrm{ZY}$; Writing-Original Draft Preparation, Y-LZ; Writing-Review and Editing, GS.

\section{FUNDING}

This work was supported by the National Natural Science Foundation of China (81871844); Shuguang Program supported by Shanghai Education Development Foundation and Shanghai Municipal Education Commission (18SG48); the Shanghai Key Lab of Human Performance (Shanghai University of Sport) (11DZ2261100); The scientific and technological research program of the Shanghai Science and Technology Committee (19080503100); Shanghai Frontiers Science Research Base of Exercise and Metabolic Health.

\section{SUPPLEMENTARY MATERIAL}

The Supplementary Material for this article can be found online at: https://www.frontiersin.org/articles/10.3389/fmolb.2021.780730/ full\#supplementary-material

Chen, H.-P., Zhou, W., Kang, L.-M., Yan, H., Zhang, L., Xu, B.-H., et al. (2014). Intrathecal miR-96 Inhibits Nav1.3 Expression and Alleviates Neuropathic Pain in Rat Following Chronic Construction Injury. Neurochem. Res. 39, 76-83. doi:10.1007/s11064-013-1192-z

Chen, J., Li, C., Liu, W., Yan, B., Hu, X., and Yang, F. (2019). miRNA-155 Silencing Reduces Sciatic Nerve Injury in Diabetic Peripheral Neuropathy. J. Mol. Endocrinol. 63, 227-238. doi:10.1530/jme-19-0067

Chen, P., Wang, C., Lin, D., Li, B., Ye, S., Qu, J., et al. (2021). Identification of Slc6a19os and SOX11 as Two Novel Essential Genes in Neuropathic Pain Using Integrated Bioinformatic Analysis and Experimental Verification. Front. Neurosci. 15, 627945. doi:10.3389/fnins.2021.627945

Cohen, S. P., and Mao, J. (2014). Neuropathic Pain: Mechanisms and Their Clinical Implications. BMJ 348, f7656. doi:10.1136/bmj.f7656

Costigan, M., Scholz, J., and Woolf, C. J. (2009). Neuropathic Pain: a Maladaptive Response of the Nervous System to Damage. Annu. Rev. Neurosci. 32, 1-32. doi:10.1146/annurev.neuro.051508.135531

Davies, A., Hendrich, J., Van Minh, A. T., Wratten, J., Douglas, L., and Dolphin, A. C. (2007). Functional Biology of the $a 2 \delta$ Subunits of Voltage-Gated Calcium Channels. Trends Pharmacol. Sci. 28, 220-228. doi:10.1016/j.tips.2007.03.005

Dolphin, A. C. (2018). Voltage-gated Calcium Channel Alpha 2delta Subunits: an Assessment of Proposed Novel Roles. F1000Res 7, 1830. doi:10.12688/ f1000research.16104.1

Dong, Y., Li, P., Ni, Y., Zhao, J., and Liu, Z. (2014). Decreased MicroRNA-125a-3p Contributes to Upregulation of P38 MAPK in Rat Trigeminal Ganglions with Orofacial Inflammatory Pain. Plos One 9, e111594. doi:10.1371/ journal.pone.0111594 
Duan, Z., Zhang, J., Li, J., Pang, X., and Wang, H. (2020). Inhibition of microRNA155 Reduces Neuropathic Pain during Chemotherapeutic Bortezomib via Engagement of Neuroinflammation. Front. Oncol. 10, 416. doi:10.3389/ fonc. 2020.00416

Elramah, S., López-González, M. J., Bastide, M., Dixmérias, F., Roca-Lapirot, O., Wielanek-Bachelet, A. C., et al. (2017). Spinal miRNA-124 Regulates Synaptopodin and Nociception in an Animal Model of Bone Cancer Pain. Sci. Rep. 7, 10949. doi:10.1038/s41598-017-10224-1

Favereaux, A., Thoumine, O., Bouali-Benazzouz, R., Roques, V., Papon, M.-A., Salam, S. A., et al. (2011). Bidirectional Integrative Regulation of Cav1.2 Calcium Channel by microRNA miR-103: Role in Pain. EMBO J. 30, 3830-3841. doi:10.1038/emboj.2011.249

Feng, Y., Chen, L., Luo, Q., Wu, M., Chen, Y., and Shi, X. (2018). Involvement of microRNA-146a in Diabetic Peripheral Neuropathy through the Regulation of Inflammation. Dddt 12, 171-177. doi:10.2147/dddt.s157109

Gandla, J., Lomada, S. K., Lu, J., Kuner, R., and Bali, K. K. (2017). miR-34c-5p Functions as Pronociceptive microRNA in Cancer Pain by Targeting Cav2.3 Containing Calcium Channels. Pain 158, 1765-1779. doi:10.1097/ j.pain.0000000000000971

Guo, J. B., Zhu, Y., Chen, B. L., Song, G., Peng, M. S., Hu, H. Y., et al. (2019). Network and Pathway-based Analysis of microRNA Role in Neuropathic Pain in Rat Models. J. Cel Mol Med 23, 4534-4544. doi:10.1111/ jcmm. 14357

Hamada, A., Yamashita, A., Suhara, Y., Narita, M., Tsuyama, J., Kuzumaki, N., et al. (2014). Micro-RNA 200b/429 in the Nucleus Accumbens Plays a Key Role in the Neuropathic Pain. J. Pharmacol. Sci. 124, 101P.

Huang, Z.-Z., Wei, J.-Y., Ou-Yang, H.-D., Li, D., Xu, T., Wu, S.-L., et al. (2016). Mir-500-mediated GAD67 Downregulation Contributes to Neuropathic Pain. J. Neurosci. 36, 6321-6331. doi:10.1523/jneurosci.0646-16.2016

Ito, N., Sakai, A., Miyake, N., Maruyama, M., Iwasaki, H., Miyake, K., et al. (2017). miR15b Mediates Oxaliplatin-Induced Chronic Neuropathic Pain through BACE1 Down-Regulation. Br. J. Pharmacol. 174, 386-395. doi:10.1111/bph.13698

Jenuwein, T., and Allis, C. D. (2001). Translating the Histone Code. Science 293, 1074-1080. doi:10.1126/science.1063127

Jiang, B.-C., Cao, D.-L., Zhang, X., Zhang, Z.-J., He, L.-N., Li, C.-H., et al. (2016). CXCL13 Drives Spinal Astrocyte Activation and Neuropathic Pain via CXCR5. J. Clin. Invest. 126, 745-761. doi:10.1172/jci81950

Koundouros, N., Karali, E., Tripp, A., Valle, A., Inglese, P., Perry, N. J. S., et al. (2020). Metabolic Fingerprinting Links Oncogenic PIK3CA with Enhanced Arachidonic Acid-Derived Eicosanoids. Cell 181, 1596-1611. doi:10.1016/ j.cell.2020.05.053

Li, L., Shao, J., Wang, J., Liu, Y., Zhang, Y., Zhang, M., et al. (2019). MiR-30b-5p Attenuates Oxaliplatin-Induced Peripheral Neuropathic Pain through the Voltage-Gated Sodium Channel Nav1.6 in Rats. Neuropharmacology 153, 111-120. doi:10.1016/j.neuropharm.2019.04.024

Li, T., Wan, Y., Sun, L., Tao, S., Chen, P., Liu, C., et al. (2019). Inhibition of microRNA-15a/16 Expression Alleviates Neuropathic Pain Development through Upregulation of G Protein-Coupled Receptor Kinase 2. Biomolecules Ther. 27, 414-422. doi:10.4062/biomolther.2018.073

Li, X., Wang, S., Yang, X., and Chu, H. (2021). miR-142-3p Targets AC9 to Regulate Sciatic Nerve Injury-induced N-europathic P-ain by R-egulating the cAMP/AMPK S-ignalling P-athway. Int. J. Mol. Med. 47, 561-572. doi:10.3892/ ijmm.2020.4824

Lin, C.-R., Chen, K.-H., Yang, C.-H., Huang, H.-W., and Sheen-Chen, S.-M. (2014). Intrathecal miR-183 Delivery Suppresses Mechanical Allodynia in Mononeuropathic Rats. Eur. J. Neurosci. 39, 1682-1689. doi:10.1111/ejn.12522

Liu, C., Yang, J., Liu, H., Xia, T., and Zhang, F. (2020). miR-300 Mitigates CancerInduced Bone Pain through Targeting HMGB1 in Rat Models. Genes Genom 42, 309-316. doi:10.1007/s13258-019-00904-9

Liu, L., Xu, D., Wang, T., Zhang, Y., Yang, X., Wang, X., et al. (2020). Epigenetic Reduction of miR-214-3p Upregulates Astrocytic colony-stimulating Factor-1 and Contributes to Neuropathic Pain Induced by Nerve Injury. Pain 161, 96-108. doi:10.1097/j.pain.0000000000001681

Liu, Y., Latremoliere, A., Li, X., Zhang, Z., Chen, M., Wang, X., et al. (2018). Touch and Tactile Neuropathic Pain Sensitivity Are Set by Corticospinal Projections. Nature 561, 547-550. doi:10.1038/s41586-018-0515-2
López-González, M. J., Landry, M., and Favereaux, A. (2017). MicroRNA and Chronic Pain: From Mechanisms to Therapeutic Potential. Pharmacol. Ther. 180, 1-15. doi:10.1016/j.pharmthera.2017.06.001

Lu, S., Ma, S., Wang, Y., Huang, T., Zhu, Z., and Zhao, G. (2017). Mus MusculusmicroRNA-449a Ameliorates Neuropathic Pain by Decreasing the Level of KCNMA1 and TRPA1, and Increasing the Level of TPTE. Mol. Med. Rep. 16, 353-360. doi:10.3892/mmr.2017.6559

Maekawa, Y., Minato, Y., Ishifune, C., Kurihara, T., Kitamura, A., Kojima, H., et al. (2008). Notch2 Integrates Signaling by the Transcription Factors RBP-J and CREB1 to Promote T Cell Cytotoxicity. Nat. Immunol. 9, 1140-1147. doi:10.1038/ni.1649

Manners, M. T., Ertel, A., Tian, Y., and Ajit, S. K. (2016). Genome-wide Redistribution of MeCP2 in Dorsal Root Ganglia after Peripheral Nerve Injury. Epigenetics \& Chromatin 9, 23. doi:10.1186/s13072-016-0073-5

Mathieu, M.-E., Faucheux, C., Saucourt, C., Soulet, F., Gauthereau, X., Fédou, S., et al. (2013). MRAS GTPase Is a Novel Stemness Marker that Impacts Mouse Embryonic Stem Cell Plasticity and Xenopus Embryonic Cell Fate. Development 140, 3311-3322. doi:10.1242/dev.091082

Miao, F., Wang, R., Cui, G., Li, X., Wang, T., and Li, X. (2019). Engagement of MicroRNA-155 in Exaggerated Oxidative Stress Signal and TRPA1 in the Dorsal Horn of the Spinal Cord and Neuropathic Pain during Chemotherapeutic Oxaliplatin. Neurotox Res. 36, 712-723. doi:10.1007/ s12640-019-00039-5

Pan, Z., Shan, Q., Gu, P., Wang, X. M., Tai, L. W., Sun, M., et al. (2018). miRNA23a/CXCR4 Regulates Neuropathic Pain via Directly Targeting TXNIP/NLRP3 Inflammasome axis. J. Neuroinflammation 15, 29. doi:10.1186/s12974-0181073-0

Peirs, C., and Seal, R. P. (2016). Neural Circuits for Pain: Recent Advances and Current Views. Science 354, 578-584. doi:10.1126/science.aaf8933

Penas, C., and Navarro, X. (2018). Epigenetic Modifications Associated to Neuroinflammation and Neuropathic Pain after Neural Trauma. Front. Cel. Neurosci. 12, 158. doi:10.3389/fncel.2018.00158

Peng, C., Li, L., Zhang, M.-D., Bengtsson Gonzales, C., Parisien, M., Belfer, I., et al. (2017). miR-183 Cluster Scales Mechanical Pain Sensitivity by Regulating Basal and Neuropathic Pain Genes. Science 356, 1168-1171. doi:10.1126/ science.aam7671

Pope, J. E., Deer, T. R., and Kramer, J. (2013). A Systematic Review: Current and Future Directions of Dorsal Root Ganglion Therapeutics to Treat Chronic Pain. Pain Med. 14, 1477-1496. doi:10.1111/pme.12171

Qiu, S., Liu, B., Mo, Y., Wang, X., Zhong, L., Han, X., et al. (2020). MiR-101 Promotes Pain Hypersensitivity in Rats with Chronic Constriction Injury via the MKP-1 Mediated MAPK Pathway. J. Cel Mol Med 24, 8986-8997. doi: $10.1111 /$ jcmm. 15532

Rodón, L., Gonzàlez-Juncà, A., del Mar Inda, M., Sala-Hojman, A., Martínez-Sáez, E., and Seoane, J. (2014). Active CREB1 Promotes a Malignant TGF $\beta 2$ Autocrine Loop in Glioblastoma. Cancer Discov. 4, 1230-1241. doi:10.1158/ 2159-8290.cd-14-0275

Shannon, P., Markiel, A., Ozier, O., Baliga, N. S., Wang, J. T., Ramage, D., et al. (2003). Cytoscape: a Software Environment for Integrated Models of Biomolecular Interaction Networks. Genome Res. 13, 2498-2504. doi:10.1101/gr.1239303

Shao, J., Cao, J., Wang, J., Ren, X., Su, S., Li, M., et al. (2016). MicroRNA-30b Regulates Expression of the Sodium Channel Nav1.7 in Nerve Injury-Induced Neuropathic Pain in the Rat. Mol. Pain 12, 1-13. doi:10.1177/ 1744806916671523

Shi, J., Jiang, K., and Li, Z. (2018). MiR-145 Ameliorates Neuropathic Pain via Inhibiting Inflammatory Responses and mTOR Signaling Pathway by Targeting Akt3 in a Rat Model. Neurosci. Res. 134, 10-17. doi:10.1016/ j.neures.2017.11.006

Simeoli, R., Montague, K., Jones, H. R., Castaldi, L., Chambers, D., Kelleher, J. H., et al. (2017). Exosomal Cargo Including microRNA Regulates Sensory Neuron to Macrophage Communication after Nerve Trauma. Nat. Commun. 8, 1778. doi:10.1038/s41467-017-01841-5

Son, S. M., Park, S. J., Lee, H., Siddiqi, F., Lee, J. E., Menzies, F. M., et al. (2019). Leucine Signals to mTORC1 via its Metabolite Acetyl-Coenzyme A. Cel Metab. 29, 192-201. doi:10.1016/j.cmet.2018.08.013 
Song, G., Yang, Z., Guo, J., Zheng, Y., Su, X., and Wang, X. (2020). Interactions Among lncRNAs/circRNAs, miRNAs, and mRNAs in Neuropathic Pain. Neurotherapeutics 17, 917-931. doi:10.1007/s13311-020-00881-y

Su, S., Shao, J., Zhao, Q., Ren, X., Cai, W., Li, L., et al. (2017). MiR-30b Attenuates Neuropathic Pain by Regulating Voltage-Gated Sodium Channel Nav1.3 in Rats. Front. Mol. Neurosci. 10, 126. doi:10.3389/fnmol.2017.00126

Sun, L., Xia, R., Jiang, J., Wen, T., Huang, Z., Qian, R., et al. (2021). MicroRNA-96 Is Required to Prevent Allodynia by Repressing Voltage-Gated Sodium Channels in Spinal Cord. Prog. Neurobiol. 202, 102024. doi:10.1016/ j.pneurobio.2021.102024

Tan, M., Shen, L., and Hou, Y. (2020). Epigenetic Modification of BDNF Mediates Neuropathic Pain via miR-30a-3p/EP300 axis in CCI Rats. Biosci. Rep. 40, BSR20194442. doi:10.1042/BSR20194442

Tang, S., Jing, H., Song, F., Huang, H., Li, W., Xie, G., et al. (2021). MicroRNAs in the Spinal Microglia Serve Critical Roles in Neuropathic Pain. Mol. Neurobiol. 58, 132-142. doi:10.1007/s12035-020-02102-1

Tramullas, M., Francés, R., De La Fuente, R., Velategui, S., Carcelén, M., García, R., et al. (2018). MicroRNA-30c-5p Modulates Neuropathic Pain in Rodents. Sci. Transl Med. 10, eaao6299. doi:10.1126/scitranslmed.aao6299

Tsuda, M., Koga, K., Chen, T., and Zhuo, M. (2017). Neuronal and Microglial Mechanisms for Neuropathic Pain in the Spinal Dorsal Horn and Anterior Cingulate Cortex. J. Neurochem. 141, 486-498. doi:10.1111/jnc.14001

Wang, Z., Liu, F., Wei, M., Qiu, Y., Ma, C., Shen, L., et al. (2018). Chronic Constriction Injury-Induced microRNA-146a-5p Alleviates Neuropathic Pain through Suppression of IRAK1/TRAF6 Signaling Pathway. J. Neuroinflammation 15, 179. doi:10.1186/s12974-018-1215-4

Wen, J., He, T., Qi, F., and Chen, H. (2019). MiR-206-3p Alleviates Chronic Constriction Injury-Induced Neuropathic Pain through Targeting HDAC4. Exp. Anim. 68, 213-220. doi:10.1538/expanim.18-0091

Wu, B., Guo, Y., Chen, Q., Xiong, Q., and Min, S. (2019). MicroRNA-193a Downregulates HMGB1 to Alleviate Diabetic Neuropathic Pain in a Mouse Model. Neuroimmunomodulation 26, 250-257. doi:10.1159/000503325

Wu, X. P., Yang, Y. P., She, R. X., Xing, Z. M., Chen, H. W., and Zhang, Y. W. (2019). microRNA-329 Reduces Bone Cancer Pain through the LPAR1dependent LPAR1/ERK Signal Transduction Pathway in Mice. Ther. Adv. Med. Oncol. 11, 1758835919875319. doi:10.1177/1758835919875319

Wu, Y., Gu, Y., and Shi, B. (2020). miR-590-3p Alleviates Diabetic Peripheral Neuropathic Pain by Targeting RAP1A and Suppressing Infiltration by the T Cells. Acta Biochim. Pol. 67, 587-593. doi:10.18388/abp.2020_5451

Xie, T., Zhang, J., Kang, Z., Liu, F., and Lin, Z. (2020). miR-101 DownRegulates mTOR Expression and Attenuates Neuropathic Pain in Chronic Constriction Injury Rat Models. Neurosci. Res. 158, 30-36. doi:10.1016/ j.neures.2019.09.002

Xu, B., Cao, J., Zhang, J., Jia, S., Wu, S., Mo, K., et al. (2017). Role of MicroRNA-143 in Nerve Injury-Induced Upregulation of Dnmt3a Expression in Primary Sensory Neurons. Front. Mol. Neurosci. 10, 350. doi:10.3389/fnmol.2017.00350

Yan, J., Yu, H., Shen, J., Han, C., Li, C., Shen, X., et al. (2020a). Early Overexpressing of microRNA-145 Effectively Precludes the Development of Neuropathic Mechanical Hyperalgesia via Suppressing Nav1.8 in Diabetic Rats. Pain Physician 23, E673-e686.

Yan, J., Yu, H., Shen, J., Han, C., Li, C., Shen, X., et al. (2020b). Early Overexpressing of microRNA-145 Effectively Precludes the Development of Neuropathic Mechanical Hyperalgesia via Suppressing Nav1.8 in Diabetic Rats. Pain Physician 23, E673-e686.

Yan, X. T., Xu, Y., Cheng, X. L., He, X. H., Wang, Y., Zheng, W. Z., et al. (2019). SP1, MYC, CTNNB1, CREB1, JUN Genes as Potential Therapy Targets for Neuropathic Pain of Brain. J. Cel Physiol 234, 6688-6695. doi:10.1002/jcp.27413

Yang, D., Yang, Q., Wei, X., Liu, Y., Ma, D., Li, J., et al. (2017). The Role of miR190a-5p Contributes to Diabetic Neuropathic Pain via Targeting SLC17A6. Jpr 10, 2395-2403. doi:10.2147/jpr.s133755

Yang, F. R., Chen, J., Yi, H., Peng, L. Y., Hu, X. L., and Guo, Q. L. (2019). MicroRNA-7a Ameliorates Neuropathic Pain in a Rat Model of Spinal Nerve Ligation via the Neurofilament Light Polypeptide-dependent Signal Transducer and Activator of Transcription Signaling Pathway. Mol. Pain 15, 1744806919842464. doi:10.1177/1744806919842464
Ye, G., Zhang, Y., Zhao, J., Chen, Y., Kong, L., Sheng, C., et al. (2020). miR-384-5p Ameliorates Neuropathic Pain by Targeting SCN3A in a Rat Model of Chronic Constriction Injury. Neurol. Res. 42, 299-307. doi:10.1080/ 01616412.2020 .1723313

Yeh, T. Y., Luo, I. W., Hsieh, Y. L., Tseng, T. J., Chiang, H., and Hsieh, S. T. (2020). Peripheral Neuropathic Pain: From Experimental Models to Potential Therapeutic Targets in Dorsal Root Ganglion Neurons. Cells 9, 2725. doi: $10.3390 /$ cells 9122725

Zhang, H., and Chen, H. (2021). TRPA1 Involved in miR-141-5p-Alleviated Neuropathic Pain Induced by Oxaliplatin. NeuroReport 32, 284-290. doi:10.1097/wnr.0000000000001589

Zhang, J., Rong, L., Shao, J., Zhang, Y., Liu, Y., Zhao, S., et al. (2021). Epigenetic Restoration of Voltage-gated Potassium Channel Kv1.2 Alleviates Nerve Injury-induced Neuropathic Pain. J. Neurochem. 156, 367-378. doi:10.1111/ jnc. 15117

Zhang, X., Guo, H., Xie, A., Liao, O., Ju, F., and Zhou, Y. (2020). MicroRNA-144 Relieves Chronic Constriction Injury-induced Neuropathic Pain via Targeting RASA1. Biotechnol. Appl. Biochem. 67, 294-302. doi:10.1002/bab.1854

Zhang, Y., Mou, J., Cao, L., Zhen, S., Huang, H., Bao, H., et al. (2018). MicroRNA142-3p Relieves Neuropathic Pain by Targeting High Mobility Group Box 1. Int. J. Mol. Med. 41, 501-510. doi:10.3892/ijmm.2017.3222

Zhang, Y.-H., Hu, H.-Y., Xiong, Y.-C., Peng, C., Hu, L., Kong, Y.-Z., et al. (2021). Exercise for Neuropathic Pain: A Systematic Review and Expert Consensus. Front. Med. 8, 756940. doi:10.3389/fmed.2021.756940

Zhang, Y., Chen, Q., Nai, Y., and Cao, C. (2020). Suppression of miR-155 Attenuates Neuropathic Pain by Inducing an M1 to M2 Switch in Microglia. fn 58, 70-82. doi:10.5114/fn.2020.94008

Zhang, Y., Liu, J., Wang, X., Zhang, J., and Xie, C. (2021). Extracellular Vesicle-Encapsulated microRNA-23a from Dorsal Root Ganglia Neurons Binds to A20 and Promotes Inflammatory Macrophage Polarization Following Peripheral Nerve Injury. Aging 13, 6752-6764. doi:10.18632/ aging. 202532

Zheng, Y.-L., Guo, J.-B., Song, G., Yang, Z., Su, X., Chen, P.-J., et al. (2021). The Role of Circular RNAs in Neuropathic Pain. Neurosci. Biobehavioral Rev. doi:10.1016/j.neubiorev.2021.10.048

Zhong, L., Xiao, W., Wang, F., Liu, J., and Zhi, L. J. (2019). miR-21-5p Inhibits Neuropathic Pain Development via Directly Targeting C-C Motif Ligand 1 and Tissue Inhibitor of Metalloproteinase-3. J. Cel Biochem 120, 16614-16623. doi: $10.1002 /$ jcb. 28920

Zhou, J., Zhuang, T., Ma, P., Shan, L., Sun, X. D., Gong, S., et al. (2020). MicroRNA547-5p-mediated Interleukin-33/suppressor of Tumorigenicity 2 Signaling Underlies the Genesis and Maintenance of Neuropathic Pain and Is Targeted by the Therapy with Bone Marrow Stromal Cells. Mol. Pain 16, 1744806920931737. doi:10.1177/1744806920931737

Zhu, H., Xue, C., Yao, M., Wang, H., Zhang, P., Qian, T., et al. (2018). miR-129 Controls Axonal Regeneration via Regulating Insulin-like Growth Factor-1 in Peripheral Nerve Injury. Cell Death Dis 9, 720. doi:10.1038/s41419-018-0760-1

Conflict of Interest: The authors declare that the research was conducted in the absence of any commercial or financial relationships that could be construed as a potential conflict of interest.

Publisher's Note: All claims expressed in this article are solely those of the authors and do not necessarily represent those of their affiliated organizations, or those of the publisher, the editors, and the reviewers. Any product that may be evaluated in this article, or claim that may be made by its manufacturer, is not guaranteed or endorsed by the publisher.

Copyright (c) 2022 Zheng, Su, Chen, Guo, Song, Yang, Chen and Wang. This is an open-access article distributed under the terms of the Creative Commons Attribution License (CC BY). The use, distribution or reproduction in other forums is permitted, provided the original author(s) and the copyright owner(s) are credited and that the original publication in this journal is cited, in accordance with accepted academic practice. No use, distribution or reproduction is permitted which does not comply with these terms. 